\title{
ON THE ORDER AND LOWER ORDER OF ENTIRE FUNCTIONS WITH RADIALLY DISTRIBUTED ZEROS
}

\author{
NORBERT STEINMETZ
}

\begin{abstract}
It is shown that the order and lower order of an entire function with zeros restricted to $k$ distinct rays differ at mcst by $k$, if either $k \leqslant 2$ or if the zeros or the rays are regularly distributed.
\end{abstract}

1. Introduction. Throughout this paper $f$ will denote an entire function whose zeros are restricted to $k$ distinct rays $\arg z=\omega_{j}\left(0 \leqslant \omega_{1}<\omega_{2}<\cdots<\omega_{k}<2 \pi\right)$. The order and lower order of $f$ are defined to be

$$
\begin{aligned}
& \lambda(f) \\
& \mu(f)
\end{aligned}=\lim \sup _{\inf } \frac{\log T(r, f)}{\log r} \quad(r \rightarrow \infty) .
$$

By $N(r)$ and $N_{j}(r)$ we denote the integrated counting functions of all zeros of $f$ and of those on $\arg z=\omega_{j}$, respectively.

It was proved by Edrei and Fuchs [2] (see also $[1,3,4,6]$ ) that the order and the lower order are cofinite. But, in general, no explicit upper bound for $\lambda(f)$ is known. Edrei and Fuchs [2] constructed an entire function $F$ with real negative zeros of prescribed order $\lambda$ and lower order $\mu$, subject only to $0<\mu \leqslant \lambda<1$. Therefore, the least upper bound for $\lambda(f)$ in terms of $\mu(f)$ and $k$, if there is any, is at least $[\mu(f)]+k$, where [ ] denotes the greatest integer function (consider $f(z)=F\left(z^{k}\right), k$ a positive integer). The inequality

$$
\lambda(f) \leqslant[\mu(f)]+k
$$

is known to be true for $k=1$ (see $[1,3,6])$. It will be shown that (1) is valid for $k=2$, too, and also, for general $k$, if the rays $\arg z=\omega$, or the zeros of $f$ are regularly distributed in some sense.

2. Statement of results. There is no loss of generality in assuming $f(0)=1$. If $f$ has finite lower order, let $q$ be the smallest integer such that

$$
\liminf _{r \rightarrow \infty} \frac{T(r, f)}{r^{q+1}}<\infty
$$

Clearly, $q \leqslant \mu(f) \leqslant q+1$.

Received by the editors June 10. 1982.

1980 Mathematics Subject Classification. Primary 30D15: Secondary 30D20.

Key words and phrases. Order, lower order of an entire function, distribution of zeros.

(5) 1983 American Mathematical Society 0002-9939/82/0000)-0927/\$01.75 
All our results will be derived from the following

THEOREM. Under the hypotheses stated above there exists a sequence $r_{n} \uparrow \infty$ such that, for any integer $p, p>q$,

$$
\sum_{j=1}^{k} e^{i p \omega,} \int_{0}^{r_{n}} \frac{N_{j}(t)}{t^{p+1}} d t=O(1)
$$

as $n \rightarrow \infty$.

Corollary 1. $\mu(f)<\infty$ implies $\lambda(f)<\infty$.

Corollary 2. $k=1$ and $\mu(f)<\infty$ imply $\lambda(f) \leqslant q+1 \leqslant[\mu(f)]+1$.

COROLlaRY 3. $k=2$ and $\mu(f)<\infty$ imply either $\lambda(f) \leqslant q+1$ or $q+1<\lambda(f)$ $\leqslant q+2 \leqslant[\mu(f)]+2$. In this case, $\omega_{2}-\omega_{1}=(2 s+1) \pi /(q+1)(s, 0 \leqslant s \leqslant q$, an integer).

REMARK. In [1] Abi-Khuzam proved for $k=2, \omega_{1}=0, \omega_{2}=m \pi / \alpha$ ( $m$ and $\alpha$ relative prime positive integers): $\lambda(f) \leqslant[\mu(f)]+\alpha$ if $m$ is even, and $\lambda(f) \leqslant[\mu(f)]$ $+2 \alpha$ if $m$ is odd. As Corollary 3 shows, this is only sharp if $\alpha=m=1$, i.e. if $f$ has only real zeros (see also Volkmann [6]).

CoROllaRY 4. If $\omega_{j}-\omega_{j-1}=2 \pi / k\left(1 \leqslant j \leqslant k ; \omega_{0}=\omega_{k}-2 \pi\right)$, then $\mu(f)<\infty$ implies $\lambda(f) \leqslant q+l \leqslant[\mu(f)]+k$, where $l, 1 \leqslant l \leqslant k$, is the smallest integer satisfying $q+l=k s$ ( $s$ an integer $)$.

COROllaRY 5. If the limits $\alpha_{j}:=\lim _{r \rightarrow \infty} N_{j}(r) / N(r)$ exist, then $\lambda(f) \leqslant q+k \leqslant$ $[\mu(f)]+k$.

Remark. Given $0<\alpha \leqslant \beta<1$, Edrei and Fuchs [2] constructed an entire function $F$ of lower order $\alpha$ and order $\beta$, having only negative zeros. Thus, $f(z)=F\left(-z^{k}\right)$ has lower order $\mu=k \alpha$ and order $\lambda=k \beta$, which may be chosen arbitrarily near to $[\mu]+k$. The zeros of $f$ are regularly distributed on $k$ distinct rays $\arg z=2 \pi j / k$ $(0 \leqslant j<k)$, and so this example does not only prove that inequality (1) is sharp for every $k$ (if it is true in general), but also the sharpness of the statements of Corollaries 2-5.

3. Proof of the Theorem. Let $a_{1}, a_{2}, \ldots$ be the zeros of $f$ repeated according to multiplicity and assume $\log f(z)=c_{1} z+c_{2} z^{2}+\cdots$ near $z=0$. Then for any integer $p=0,1,2, \ldots$

$$
\frac{1}{2 \pi} \int_{0}^{2 \pi} \log \left|f\left(r e^{i \theta}\right)\right| e^{-i p \theta} d \theta=\frac{c_{p}}{2} r^{p}+\frac{1}{2 p} \sum_{\left|a_{\nu}\right| \leqslant r}\left(\left(\frac{r}{a_{\nu}}\right)^{p}-\left(\frac{\bar{a}_{\nu}}{r}\right)^{p}\right)
$$

(see F. Nevanlinna [5]). Clearly,

$$
\left|\frac{1}{2 \pi} \int_{0}^{2 \pi} \log \right| f\left(r e^{i \theta}\right)\left|e^{-i p \theta} d \theta\right| \leqslant 2 T(r, f)
$$


and

$$
\left|\sum_{\left|a_{\nu}\right| \leqslant r}\left(\frac{\bar{a}_{\nu}}{r}\right)^{p}\right| \leqslant n(r) \leqslant T(e r, f) .
$$

With obvious notation,

$$
\sum_{\substack{\left|a_{\nu}\right| \leqslant r \\ \arg a_{\nu}=\omega_{1}}} a_{\nu}^{-p}=e^{-i p \omega}, \int_{0}^{r d n_{j}(t)} \frac{t^{p}}{}
$$

Integrating by parts twice we get

$$
\int_{0}^{r} \frac{d n_{j}(t)}{t^{p}}=\frac{n_{j}(r)}{r^{p}}+p \frac{N_{j}(r)}{r^{p}}+p^{2} \int_{0}^{r} \frac{N_{j}(t)}{t^{p+1}} d t .
$$

which yields together with (3)-(6), after passing to conjugate values,

$$
\sum_{j=1}^{k} c^{i p \omega,} \int_{0}^{r} \frac{N_{j}(t)}{t^{p+1}} d t=O\left(\frac{T(e r, f)}{r^{p}}+1\right) \quad \text { as } r \rightarrow \infty .
$$

To complete the proof, we have only to choose $r_{n} \uparrow \infty$ such that $T\left(e r_{n}, f\right) r_{n}^{-q}$ । remains bounded as $n \rightarrow \infty$.

4. Proof of the corollaries. In all cases we may replace $\lambda(f)$ by $\lambda(0, f)$ $:=\limsup _{r \rightarrow \infty} \log N(r) / \log r$, since $\lambda(0, f)<\lambda(f)$ implies $\lambda(f)=\mu(f)$. This is easily seen from $f=P e^{g}$, where $P$ is a canonical product of order $\lambda(0, f)<\lambda(f)$ (or a polynomial). Since $e^{g}$ is of regular growth, the same is true for $f$. The method gives slightly more. Instead of inequalities of type $\lambda(0, f) \leqslant b(=q+1, q+2, q+l$ and $q+k$, respectively) we will prove that $\int_{0}^{\infty} N(t) / t^{b+1} d t$ converges (implying $N(r) / r^{h}$ $\rightarrow 0$ as $r \rightarrow \infty$ ).

Proof of Corollary 1. Assume that $\int_{0}^{\infty} N(t) / t^{p+1} d t$ diverges for some $p>q$. We divide equation (2) by $\int_{0}^{r_{n}} N(t) / t^{p+1} d t$ and choose a subsequence of $r_{n}$ (still denoted by $r_{n}$ ) such that the limits

$$
\lim _{n \rightarrow \infty} \int_{0}^{r_{n}} \frac{N_{j}(t)}{t^{p+1}} d t / \int_{0}^{r_{n}} \frac{N(t)}{t^{p+1}} d t=: \alpha_{j}
$$

exist. Then we get $\Sigma_{j=1}^{k} \alpha_{j} e^{i p \omega_{j}}=0\left(\alpha_{j} \geqslant 0, \sum_{j=1}^{k} \alpha_{j}=1\right)$, which shows that the origin belongs to the convex hull of $\left\{e^{i p \omega,}: 1 \leqslant j \leqslant k\right\}$ for any such $p$. But this is not possible for every $p$, since by Weyl's equidistribution theorem [7] there exist arbitrarily large $p$ such that all $e^{i p \omega}, 1 \leqslant j \leqslant k$, belong to an open halfplane. This proves Corollary 1.

Proof of Corollary 2. We mention only that (2) implies the boundedness of $\int_{0}^{r} N(t) / t^{q+2} d t\left(N_{1}(t) \equiv N(t)\right)$ for $r=r_{n}$ and so, by monotonicity, for any $r \geqslant 0$.

Proof of Corollary 3. If $\int_{0}^{\infty} N(t) / t^{q+2} d t$ converges, we are done. If not, both integrals $\int_{0}^{\infty} N_{j}(t) / t^{q+2} d t, j=1,2$, must diverge. Thus, dividing by $\int_{0}^{r_{n}} N_{2}(t) / t^{q+2} d t$ and letting $n$ tend to infinity, (2) gives $-e^{i(q+1)\left(\omega_{2}-\omega_{1}\right)}=1$ and so $\omega_{2}-\omega_{1}=$ $(2 s+1) \pi /(q+1), s$ an integer. In the same way, $\int_{0}^{\infty} N(t) / t^{q+3} d t=+\infty$ would imply $\omega_{2}-\omega_{1}=\left(2 s^{\prime}+1\right) \pi /(q+2)$, which is impossible. 
Proof of Corollary 4. We may assume $\omega_{j}=2(j-1) \pi / k(1 \leqslant j \leqslant k)$. If $l, 1 \leqslant l \leqslant k$, is chosen in such a way that $q+l=k s$, then for $p=q+l$ we have $e^{i p \omega_{1}}=1$ and (2) gives $\int_{0}^{r_{n}} N(t) / t^{q+1+1} d t=O(1)$, which proves Corollary 4 .

Proof of Corollary 5. We assume that $\int_{0}^{\infty} N(t) / t^{p+1} d t$ diverges for any integer $p, q<p \leqslant q+k$, and will derive a contradiction. By l'Hospital's rule we get

$$
\int_{0}^{r_{n}} \frac{N_{j}(t)}{t^{p+1}} d t / \int_{0}^{r_{n}} \frac{N(t)}{t^{p+1}} d t \rightarrow \alpha_{j} \quad \text { as } n \rightarrow \infty
$$

(even if $\alpha$, is zero). Thus, (2) gives

$$
\sum_{j=1}^{k} \alpha_{j} e^{i p \omega_{1}}=0 \quad(p=q+1, \ldots, q+k)
$$

which is impossible, since (8) has no nontrivial solution (the determinant $\left|e^{i p \omega_{1}}\right|_{j=1, \ldots k ; p=q+1 \ldots . q+k}$ does not vanish). This proves Corollary 5 .

Remark. Obviously, Corollary 5 holds true under the following weaker hypothesis: For every $p, q<p \leqslant q+k$, there exists a subsequence of $r_{n}$ (still denoted by $r_{n}$ ) such that

$$
\int_{0}^{r_{n}} \frac{N_{j}(t)}{t^{p+1}} d t / \int_{0}^{r_{n}} \frac{N(t)}{t^{p+1}} d t
$$

tends to $\alpha_{j}$ (independent of $p$ ) as $n \rightarrow \infty$.

\section{REFERENCES}

I. F. Abi-Khuzam, The order of entire functions witi radially distrihuted zeros, Proc. Amer. Math. Soc. 82 (1981). 71-75.

2. A. Edrei and W. H. J. Fuchs, On the maximum number of deficient values of certain classes of functions, Air Force Technical Report. AFOSR TN 60-402.

3. T. Kobayashi. On the lower order of an entire function. Kōdai Math. Sem. Rep. 27 (1976). 484-495.

4. J. Miles. On entire functions of infinite order with radially distributed zeros. Pacific J. Math. 31 (1979). $131-157$.

5. F. Nevanlinna, Bemerkungen zur Theorie der ganzen Funktionen endlicher Ordnung. Soc. Sci. Fenn. Comment. Phys.-Math. 4 (1923).

6. L. Volkmann, Abschätzungen für die untere Ordnung hei meromorphen Funktionen, Preprint Nr. 44 (1977), FB Mathematik, FU Berlin.

7. H. Weyl, Üher die Gleichverteilung von Zahlen mod Eins, Math. Ann. 77 (1916), 313-352.

Mathematisches Institut I, Universitat Karlsruhe, Englerstrasse 2, D-7500 Karlsruhe 1 , WEST GERMANY 